\title{
Late administration of high-frequency electrical stimulation increases nerve regeneration without aggravating neuropathic pain in a nerve crush injury
}

Hong-Lin Su ${ }^{1 \dagger}$, Chien-Yi Chiang ${ }^{2 \dagger}$, Zong-Han Lu², Fu-Chou Cheng ${ }^{3}$, Chun-Jung Chen ${ }^{3}$, Meei-Ling Sheu ${ }^{4}$, Jason Sheehan ${ }^{5}$ and Hung-Chuan Pan ${ }^{2,6^{*}}$ (D)

\begin{abstract}
Background: High-frequency transcutaneous neuromuscular electrical nerve stimulation (TENS) is currently used for the administration of electrical current in denervated muscle to alleviate muscle atrophy and enhance motor function; however, the time window (i.e. either immediate or delayed) for achieving benefit is still undetermined. In this study, we conducted an intervention of sciatic nerve crush injury using high-frequency TENS at different time points to assess the effect of motor and sensory functional recovery.

Results: Animals with left sciatic nerve crush injury received TENS treatment starting immediately after injury or 1 week later at a high frequency $(100 \mathrm{~Hz})$ or at a low frequency $(2 \mathrm{~Hz})$ as a control. In SFI gait analysis, either immediate or late admission of high-frequency electrical stimulation exerted significant improvement compared to either immediate or late administration of low-frequency electrical stimulation. In an assessment of allodynia, immediate high frequency electrical stimulation caused a significantly decreased pain threshold compared to late high-frequency or low-frequency stimulation at immediate or late time points. Immunohistochemistry staining and western blot analysis of S-100 and NF-200 demonstrated that both immediate and late high frequency electrical stimulation showed a similar effect; however the effect was superior to that achieved with low frequency stimulation. Immediate high frequency electrical stimulation resulted in significant expression of TNF- $a$ and synaptophysin in the dorsal root ganglion, somatosensory cortex, and hippocampus compared to late electrical stimulation, and this trend paralleled the observed effect on somatosensory evoked potential. The CatWalk gait analysis also showed that immediate electrical stimulation led to a significantly high regularity index. In primary dorsal root ganglion cells culture, high-frequency electrical stimulation also exerted a significant increase in expression of TNF-a, synaptophysin, and NGF in accordance with the in vivo results.
\end{abstract}

Conclusion: Immediate or late transcutaneous high-frequency electrical stimulation exhibited the potential to stimulate the motor nerve regeneration. However, immediate electrical stimulation had a predilection to develop neuropathic pain. A delay in TENS initiation appears to be a reasonable approach for nerve repair and provides the appropriate time profile for its clinical application.

Keywords: Transcutaneous electrical stimulation, Nerve regeneration, Dorsal root ganglion cell, Neuropathic pain

\footnotetext{
*Correspondence: hcpan2003@yahoo.com.tw

${ }^{\dagger}$ Hong-Lin Su and Chien-Yi Chiang contributed equally to this work.

${ }^{2}$ Department of Neurosurgery, Taichung Veterans General Hospital, 1650

Taiwan Boulevard Sec. 4, 40705 Taichung, Taiwan

Full list of author information is available at the end of the article
}

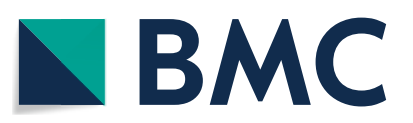

(c) The Author(s) 2018. This article is distributed under the terms of the Creative Commons Attribution 4.0 International License (http://creativecommons.org/licenses/by/4.0/), which permits unrestricted use, distribution, and reproduction in any medium, provided you give appropriate credit to the original author(s) and the source, provide a link to the Creative Commons license, and indicate if changes were made. The Creative Commons Public Domain Dedication waiver (http://creativecommons.org/ publicdomain/zero/1.0/) applies to the data made available in this article, unless otherwise stated. 


\section{Backgrounds}

Any type of nerve repair causes a period of short or long-term change in the connection between the muscle and nerve. The target muscle stayed denervated for several weeks even after immediate repair, leading to denervation-associated atrophy. A more direct method to minimize muscle atrophy is to stimulate the muscle electrically $[1,2]$. In general, short-term electrical muscle stimulation after nerve repair potentially reduces muscle atrophy $[3,4]$. Although several studies have reported the use of electrical stimulation after immediate nerve repair, the length and stimulation parameter was not adequately determined and these factors remain the subject of debate [5-9].

Neuromuscular electrical stimulation is performed by the application of electrical current directly to the skin surface and underlying muscle to induce a muscle contraction, as well as to retard muscle atrophy during the period of reinnervation [10]. In a prospective, nonrandomized trial, high-tone external muscle stimulation resulted in improvement of tingling, burning, pain, and numbness in diabetic and uremic neuropathy patients [11]. In another study, following external long-term muscle electrical stimulation in uremic neuropathy patients, physical capacity and ulnar motor conduction velocity were markedly improved [12]. To attenuate muscle atrophy and improve function of denervated muscle, stimuli should be applied several times a day at sufficient intensity, pulse duration, and frequency [13].

On the contrary, electrical stimulation can have a detrimental effect in nerve regeneration after crush injury. The transcutaneous electrical stimulation altered the morphology of axon with dark axoplasma, edema, and disorganized cytoarchitecture. In addition, a decrease in axon number was also observed with thinner myelination but with an increased number of Schwann cell nuclei [14]. Electrical stimulation reduced the muscle excitability, neural cell adhesion molecule expression, the integrity of neuromuscular junctions and muscle fiber cross sectional area $[6,8,13,15]$. Furthermore, a delay of longer than 3 months for stimulation did not increase muscle re-innervation [16]. The stimulation of a partially innervated muscle can also have adverse effects for the remaining nerves because nerve connections to the muscle are formed in an asynchronizing manner, and stimulation at this time compromised functional reinnveration [3].

The timing to start electrical stimulation is very controversial. The significant improvement in twitching tension of crushed nerve was noted only when ES was applied during the middle period (day 12-21) after nerve crush, however, no difference was observed at other time points, suggesting the stimulatory effect occurred only occurred in a specific time window [17]. ES may exert an inhibitory effect on the functional neuromuscular recovery when administrated daily while axons are renewed along the distal nerve stump but before they reach the muscle fibers [6].

Based on a previous review, several controversies exist, including the time profile for initiating neuromuscular stimulation, i.e., immediate, early or delay; electrical frequency; and intensity. In this study, animals with sciatic nerve injury were subjected to the electrical neuromuscular stimulation at the different time profiles and at different stimulation frequencies and intensities to assess the alteration in neurobehavior, electrophysiology, and the associated protein expression. In addition, a primary culture of dorsal root ganglion cells was used to investigate the response by the electrical current.

\section{Methods}

\section{Nerve crush injury model}

Male Sprague-Dawley rats weighing 250-300 g (bought from BioLASCO Taiwan Co.) were used and were anesthetized using isoflurane at $4 \%$ in the induction period and $1 \%$ in maintenance period. The gluteal splitting method was used to expose left sciatic nerve under the microscope, and the nerve was crushed using a vessel clamp $10 \mathrm{~mm}$ from the obturator [18]. The animals were randomly allocated into one of six groups as follows: Group I: sham $(n=6)$; Group II: nerve crush injury as control $(n=6)$; Group III (HFI): high-frequency $(100 \mathrm{~Hz})$ percutaneous electrical stimulation administrated immediately $(\mathrm{n}=12)$; Group IV (HFL): high-frequency $(100 \mathrm{~Hz})$ percutaneous electrical stimulation administrated 7 days after nerve crush $(n=12)$. Group V (LFI): low-frequency $(5 \mathrm{~Hz})$ percutaneous electrical stimulation administrated immediately $(\mathrm{n}=6)$; Group VI (LFL): low-frequency $(5 \mathrm{~Hz})$ percutaneous electrical stimulation administrated 7 days after nerve crush $(n=6)$. The electrical stimulation paradigm featured a treatment consisting of stimulation for $30 \mathrm{~min}$ per day for 7 consecutive days using $400 \mathrm{~ms}$ of biphasic pulses at $200 \mu \mathrm{s}$ per phase and 100 or $5 \mathrm{~Hz}$ frequency and with $6 \mathrm{~s}$ of rest (ElePulsHV-F125, Omron, Japan) [19]. Food and water were provided ad libitum before and after the operation. The animal housing environment was kept under the appropriate condition with 2 animals in a single cage, in a temperature-controlled environment at $20{ }^{\circ} \mathrm{C}$ and with alternating light and dark cycles with 12 -h intervals. After the experiment, all animals were euthanized using $\mathrm{CO} 2$. The care and operation of all animals followed the guidelines recommended by Taichung Veterans General Hospital Institutional Animal Care and Use Committee (IACUC) (Permission No.La-1061455). 


\section{Analysis of motor function recovery}

A technician blindly assessed the SFI in the various groups of animals before operation and weekly after the surgery according to our previous report $[20,21]$. Several essential parameters were taken from the footprint and all measurements were taken in the experimental and control groups. An SFI of 0 indicated normal function and -100 represented total loss of motor function.

\section{Nociceptive behaviors}

Mechanical allodynia was tested blindly by using von Frey hairs (Touch-Test Sensory Evaluator, North Coast Medical, Inc), as previously described by our group [20, $21]$. Von Frey hairs were applied in a series of grams to touch the hind paw bilaterally five times for 5-s intervals when the hind paw was placed appropriately on the platform. The withdrawal threshold was considered to be the force (gram) of the hair that caused hind-paw withdrawal in at least four out of the five applications. Thermal hyperalgesia was evaluated via a hot-plate test (Technical\& Scientific Equipment GmbH, TSE systems) according to the pervious procedure [21]. The withdrawal latency was recorded as the interval of the time from which the rat touched the $52{ }^{\circ} \mathrm{C}$ hotplate to the time of withdrawal of the paw. A maximal cut-off of twenty-seconds was used to prevent paw tissue injury.

\section{Catwalk gait analysis}

The CatWalk XT gait analysis has been previously described by our group [21]. Quantitative analysis of the data included the following parameters: step sequence distribution, regularity index (RI), print area, duration of swing and stance phases, and intensity. The data were presented as the ratio of the measurement for the left side divided by that for the right side.

\section{Evoked potential of the somatosensory cortex}

Evoked potential measurements have been previously published by our group [20, 21]. In brief, one active electrode was threaded into the dural surface of the somatosensory area $(3 \mathrm{~mm}$ lateral and $2 \mathrm{~mm}$ posterior to the bregma). Another electrode was placed as a reference over the maxillary area at approximately $20 \mathrm{~mm}$ from the active electrode. A stimulation intensity of $20 \mathrm{~mA}$ with $20-2000 \mathrm{~Hz}$ filtration was applied over the sciatic nerve $1 \mathrm{~cm}$ proximal to the injury area. The data for conduction latency and evoked potential were presented as the ratio of the measurement for the right side to the left side, to minimize the effects of anesthesia.
Isolation and cultured dorsal root ganglion cells (DRGs)

\section{subjected to electrical stimulation}

Dorsal root ganglia cells were dissected from embryonic Sprague-Dawley rat at the embryonic days 14-15 according to previous report $[20,22,23]$. The DRGs were incubated with $0.25 \%$ trypsin at $37^{\circ} \mathrm{C}$ for $15 \mathrm{~min}$ and were dissociated, washed and re-suspended with Neurobasal medium containing 2\% B27 (sigma, Inc.), $0.3 \% \mathrm{~L}$-glutamine and $100 \mathrm{ng} / \mathrm{ml}$ nerve growth factor. Finally, these cells were cultured in the dish at a density of $1 \times 10^{4}$ cells per ml of medium (16-well array station, ECIS model 800). Next, the cells were maintained and cultured in an incubator at $37^{\circ} \mathrm{C}$ and $5 \% \mathrm{CO}_{2}$. The cells were recognized by neuronal markers ( $\beta$ III tubulin) before the experimental process and were subjected to electrical stimulation for duration of $30 \mathrm{~min}$ at frequencies of 5 and $100 \mathrm{~Hz}$ with $50 \mathrm{~mA}$.

\section{Western blot analysis}

The distal end of the nerve, muscles, dorsal root ganglion cells, and brain (hippocampus/cortex) were harvested 4 weeks after the various treatment and proteins were extracted. The cell lysate of dorsal root ganglion cells after electrical stimulation were collected to determine the expression of synaptophysin, TNF- $\alpha$, and NGF. Proteins $(50 \mu \mathrm{g})$ were resolved by SDS-polyacrylamide gel electrophoresis and were transferred onto a blotting membrane [20]. After blocking with non-fat milk, the membranes were incubated with antibodies against S-100 (Neomarkers, 1:500 dilution), NF (Cellsignal, 1:1000 dilution), synaptophysin (Abcam, 1:500 dilution), TNF- $\alpha$ (Abcam, 1:1000 dilution), and NGF-R (1:1000, Abbiotec) overnight at $4{ }^{\circ} \mathrm{C}$. The intensity of the protein bands was determined by a computer image analysis system (IS1000, Alpha Innotech Corporation, CA, USA).

\section{Immunohistochemistry staining}

Dorsal root ganglion cell culture after electrical stimulation and serial 8-mm-thick section of nerve, muscle, dorsal root ganglion cells, and the brain were cut using a cryostat, and mounted on superfrost/plus slides (Menzel-Glaser, Braunschweig, Germany) were subjected to immunohistochemistry using antibodies against NGF-R (1:1000, Abbiotec), S-100(1:200, Serotec), neurofilament(1:200, Millipore), anti-synaptophysin (Abcam, 1:200 dilution), and anti-TNF- $\alpha$ (Abcam, 1:300 dilution) to detect the inflammatory response associated with nerve regeneration in sciatic nerve, dorsal root ganglion cells, and the brain. The immunoreactive signals were observed using AF 488 donkey anti-mouse IgG and AF594 donkey anti-rabbit 
(Invitrogen; 1:200 dilutions) and were then viewed using an Olympus BX40 Research Microscope.

\section{Statistical analysis}

Data are expressed as the mean \pm SE (standard error). The SFI and CatWalk data were analyzed via repeatedmeasure ANOVA followed by Bonferroni's multiple comparison method. The statistical significance of the differences among the groups was determined via oneway analysis of variance (ANOVA) followed by Dunnett's test. A $p$ value $<0.05$ was considered significant.

\section{Results}

Immediate high-frequency electrical stimulation caused significant motor function improvement but cause a predisposition to neuropathic pain

These animals were subjected to different treatments evaluated by SFI (motor function) and mechanical withdrawal threshold (sensory function), as illustrated in Fig. 1. SFI analysis demonstrated no significant improvement after low-frequency electrical stimulation with either immediate or late treatment compared to that in the control group. High-frequency stimulation, immediate electrical stimulation exerted a significant improvement as early as at day 7 with a steeper slope compared to that in the other groups. However, late electrical stimulation delayed improvement in the beginning but reached
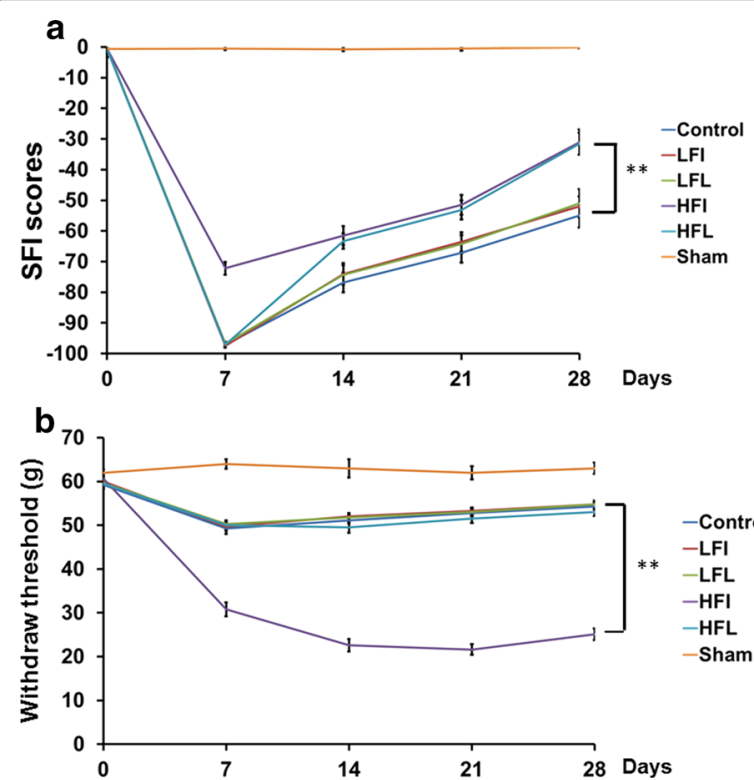

Fig. 1 Illustration of SFI and mechanical withdrawal threshold in different treatment groups at different time profiles. a Representative of SFI scores related to different time frames subjected to different treatments. $\mathbf{b}$ Representative of mechanical withdrawal threshold after different treatments given at different time points. Sham; Crush; HFI; HFL; LFI; LFL: see text. ${ }^{* *} p<0.01, \mathrm{n}=6$ the effects observed with immediate high-frequency treatment at day 14. Overall, only high-frequency stimulation-either immediate or late-showed the significant improvement in motor function compared to the control or low-frequency electrical stimulation (Fig. 1a).

In the mechanical withdrawal threshold assessment, a significant decrease in mechanical withdrawal was observed after immediate high-frequency treatment compared to that observed in the other groups. There were no significant differences in mechanical withdrawal among the control, HFL, LFI, and LFL groups (Fig. 1b). These observations suggest that immediate high-frequency electrical stimulation exerted a significant enhancement of motor function from the early period that lasted to the final point of assessment; however, this stimulation carried a higher risk of neuropathic pain. The late high-frequency electrical stimulation showed delayed improvement of motor function compared to that in the HFI group but approached the final outcome of the HFI group, without the development of neuropathic pain.

Based on the above assumption and in accordance with the guidelines of NC3Rs Animal Research: Reporting In Vivo Experiments (ARRIVE), to reduce the numbers of animals used in experimental studies, the remaining part of the study focused on determining the appropriate time profile to initiate the TENS treatment of high-frequency electrical stimulation through either immediate or late administration (Additional file 1).

\section{High-frequency electrical stimulation increased nerve myelination either immediate or late administration} For further confirmation of the nerve regeneration potential subjected to immediate or late high-frequency electrical stimulation, the sciatic nerve was harvested one month after injury. Theses nerves were subjected to immunohistochemistry analysis of S-100 and neurofilament (Fig. 2a, b). There was a significantly higher expression of myelination markers such as S-100 and neurofilament for immediate and late high-frequency electrical stimulation compared to that for the control and immediate low-frequency electrical stimulation (Fig. 2c). This result suggested that high-frequency electrical stimulation through either immediate or late administration exhibited potential for nerve regeneration.

\section{Immediate high-frequency electrical stimulation caused higher expression of the inflammatory response in the dorsal root ganglion and brain}

Synaptophysin and TNF- $\alpha$ expression in the dorsal root ganglion and the somatosensory cortex and hippocampus represents the severity of neuropathic pain. Immediate high-frequency electrical stimulation resulted in a significantly higher expression of synaptophysin and 


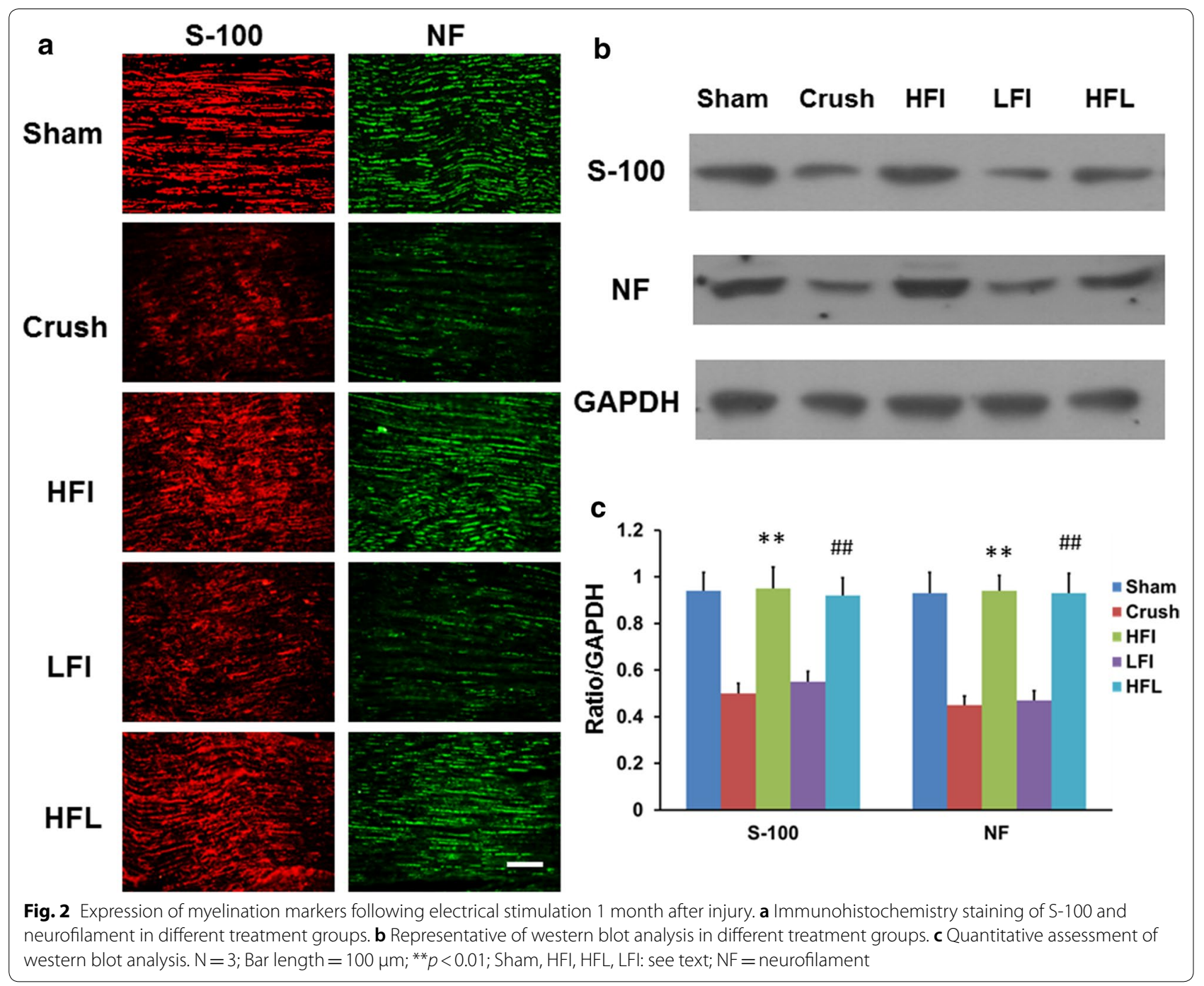

TNF- $\alpha$ in the dorsal root ganglion compared to the control treatment or high-frequency late electrical stimulation (Fig. 3a-c). Higher expression of synaptophysin and TNF- $\alpha$ in the somatosensory cortex and hippocampus was also noted in the immediate group compared to that in the late high-frequency and control groups (Fig. $4 \mathrm{a}-\mathrm{c}$ ).

\section{Alteration of CatWalk gait analysis and increased evoked potential in the somatosensory cortex after immediate high-frequency electrical stimulation}

The Catwalk gait analysis was used to examine the motor and sensory functions. Increased intensity, decreased stance, increased swing, and decreased regularity are indicative of motor function improvement. However, increased neuropathic pain produces the opposite trend. Late high-frequency electrical stimulation produces significant improvements in intensity, stance, swing, and regularity index compared to immediate high-frequency electrical stimulation or the control groups (Fig. 5a-d). This phenomenon was because the effect of immediate high-frequency electrical stimulation in motor function was compromised by the increased sensory functional impairment.

Increased evoked potential in the central nervous system is positively correlated with a peripheral nervous system injury. The increased evoked potential amplitudes in the somatosensory cortex were significantly higher after immediate high-frequency electrical stimulation than after late high-frequency electrical stimulation or the control (Fig. 6a, b). The somatosensory evoked potential data further confirmed the alteration in the neurobehavioral and histomorphological characteristics. 


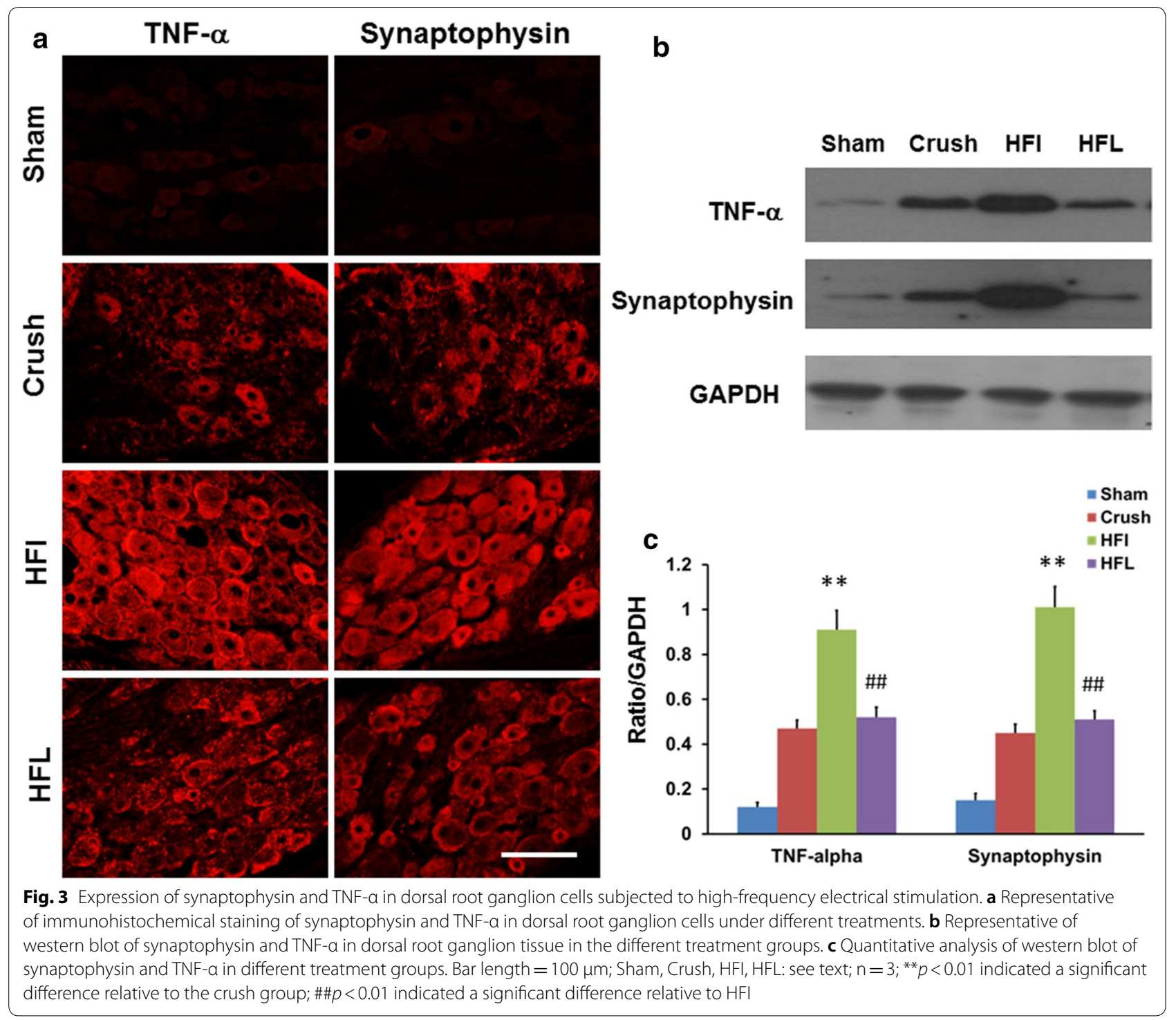

Electrical stimulation induced the expression of the inflammatory response in dorsal root ganglion cell culture

To assess the inflammatory response in dorsal root ganglion cells stimulated by direct electrical stimulation, dorsal root ganglion cells were harvested and subjected to electrical stimulation. Immunohistochemistry staining demonstrated that there was increased expression of synaptophysin, TNF- $\alpha$ and NGF-R in the dorsal root ganglion cell culture, which was related to the difference in intensity of the electrical stimulation frequency (Fig. 7a, b). Quantitative analysis demonstrated significantly higher expression of synaptophysin, TNF- $\alpha$ and NGF-R after high-frequency electrical stimulation compared to that after low-frequency electrical stimulation and the sham (Fig. 7c). Hence, high-frequency electrical stimulation harbored the potential to stimulate the dorsal root ganglion cells to express the inflammation associated proteins.

\section{Discussion}

High-frequency electrical stimulation harbored the potential to better augment nerve regeneration compared to low-frequency electrical stimulation; however, their regeneration ability was compromised by their direct electrical effect on sensory function impairment. Thus, the appropriate time profile to start the treatment after nerve injury is unclear. In this study, we found that highfrequency electrical stimulation more effectively primed dorsal root ganglion cells to express inflammatory 


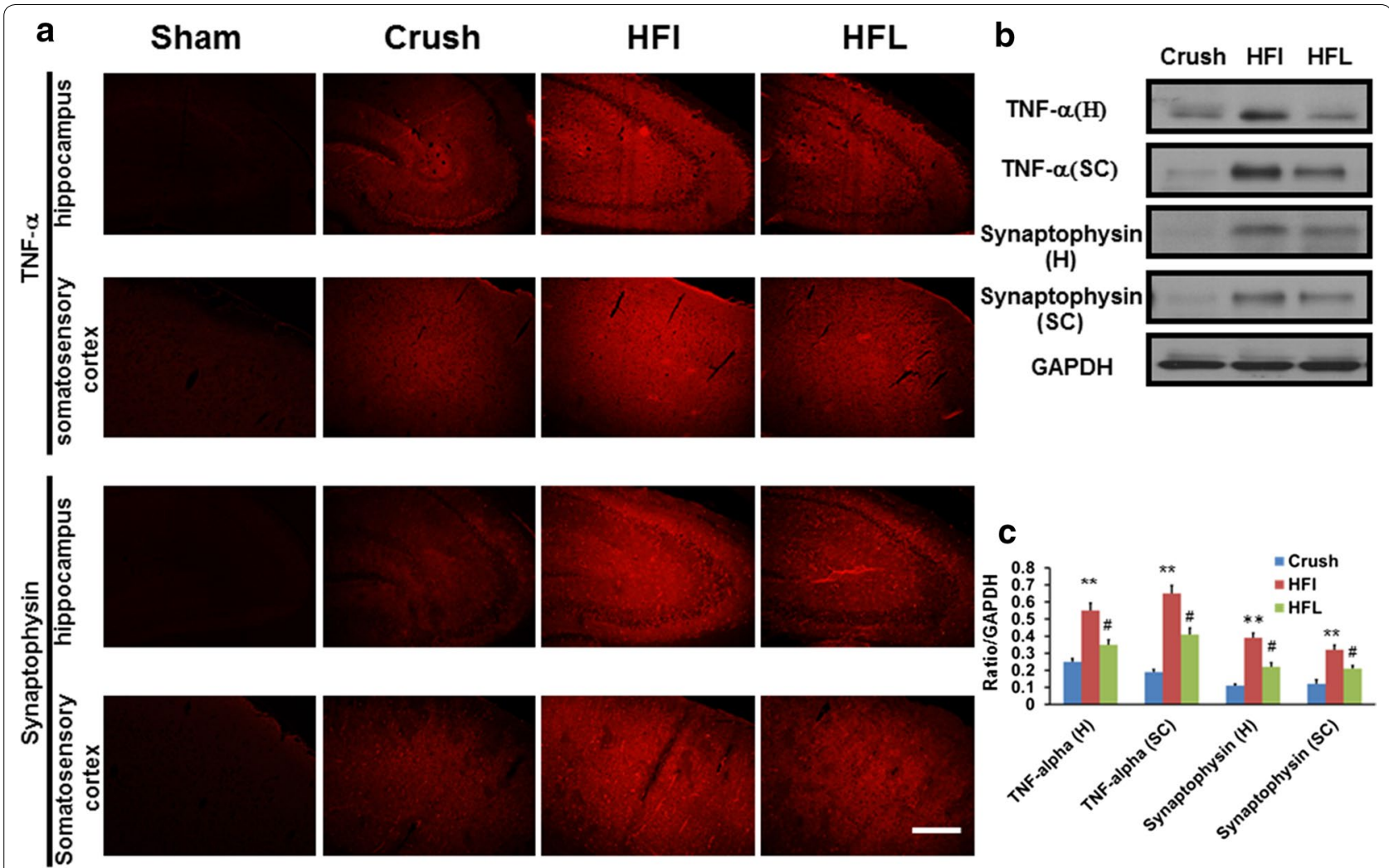

Fig. 4 Expression of synaptophysin and TNF-a in the brain after high-frequency electrical stimulation one month after injury. a Representative of synaptophysin and TNF-a in the hippocampus and somatosensory cortex in different treatment groups $\mathbf{b}$ Representative of western blot analysis of synaptophysin and TNF-a in the different treatment groups. c Quantitative analysis of western blot of synaptophysin and TNF-a for different treatment groups. Sham, Crush, HFI, HFL: see text; $N=3 ;{ }^{* *} p<0.01$ indicated the significant difference compared to crush group; \#p<0.05 indicated the significant difference compared to HFl; Bar length $=100 \mu \mathrm{m}$

cytokines such as TNF- $\alpha$, synaptophysin, and NGF. In animal studies, significantly increased nerve regeneration was noted after high-frequency electrical stimulation administrated either immediately or late; however, immediate electrical stimulation exhibited a higher potential to lead to neuropathic pain. A delay in high-frequency electrical stimulation appeared to be an appropriate time to initiate the electrical stimulation after nerve injury.

Electrical stimulation plays an important role in the treatment of neuromuscular junction disease. There are many method and types of electrical stimulation. The most common method of transcutaneous electrical stimulation used an electrical current of 90-130 Hz [24]. In another study, immediate high-frequency $(100 \mathrm{~Hz})$ electrical stimulation of the muscle exerted significantly a significant increase in the expression of neurotrophic factors, which contributed to neurological improvement [25]. In a diabetes animal study, high-frequency electrical stimulation $(200 \mathrm{~Hz})$ exerted a greater myelination effect than low-frequency stimulation $(20 \mathrm{~Hz})$ [26]. In contrast, a low-frequency percutaneous electrical stimulation of
$2 \mathrm{~Hz}$ enhanced the mean value of axonal density, blood vessel number and axon outgrowth through the nerve graft $[27,28]$. In our study, we found that high-frequency percutaneous electrical stimulation better improved the neurological outcome including neurobehavior and maturation of myelinization compared to low-frequency electrical stimulation. The results paralleled previous studies that demonstrated that high-frequency electrical stimulation contributing to better nerve regeneration potential than low-frequency electrical stimulation.

Percutaneous electrical stimulation was shown to alleviate pain in cases of musculoskeletal pain, arthritis pain, low back pain, neuropathic pain, and post-operative pain [22, 23, 29, 30]. Low-frequency electrical stimulation induced analgesia by inhibiting pain transmission through the recruitment of the descending inhibitory system; however, high-frequency $(80-100 \mathrm{~Hz})$ stimulation activated the gate control by stimulation A-beta fibers [31].There were various inconsistencies amongst previous studies with respect to the therapeutic effect according to the frequencies tested [32]. In our study, 

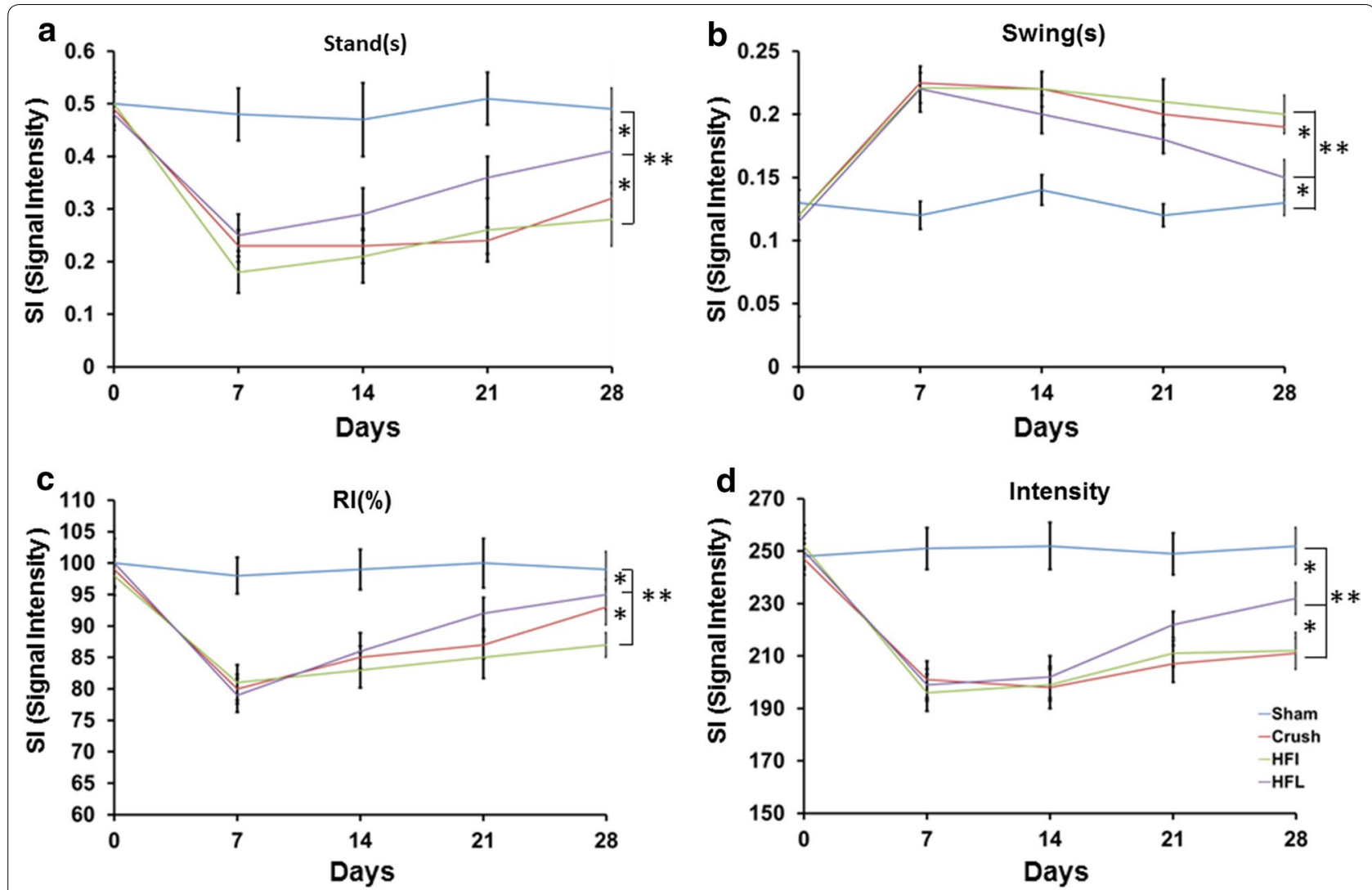

Fig. 5 CatWalk gait analysis after high-frequency electrical stimulation. a Representative of stands in different time frame subjected to different treatments. $\mathbf{b}$ Representative of swings in different time frames under different treatments. $\mathbf{c}$ Representative of RI in different time frames under different treatments. $\mathbf{d}$ Representative of hind paw intensity in different time frames under different treatments. Sham, Crush, HFI, HFL: see text; $\mathrm{N}=6$; $^{* *} p<0.01$ indicated a significant difference related to the crush group
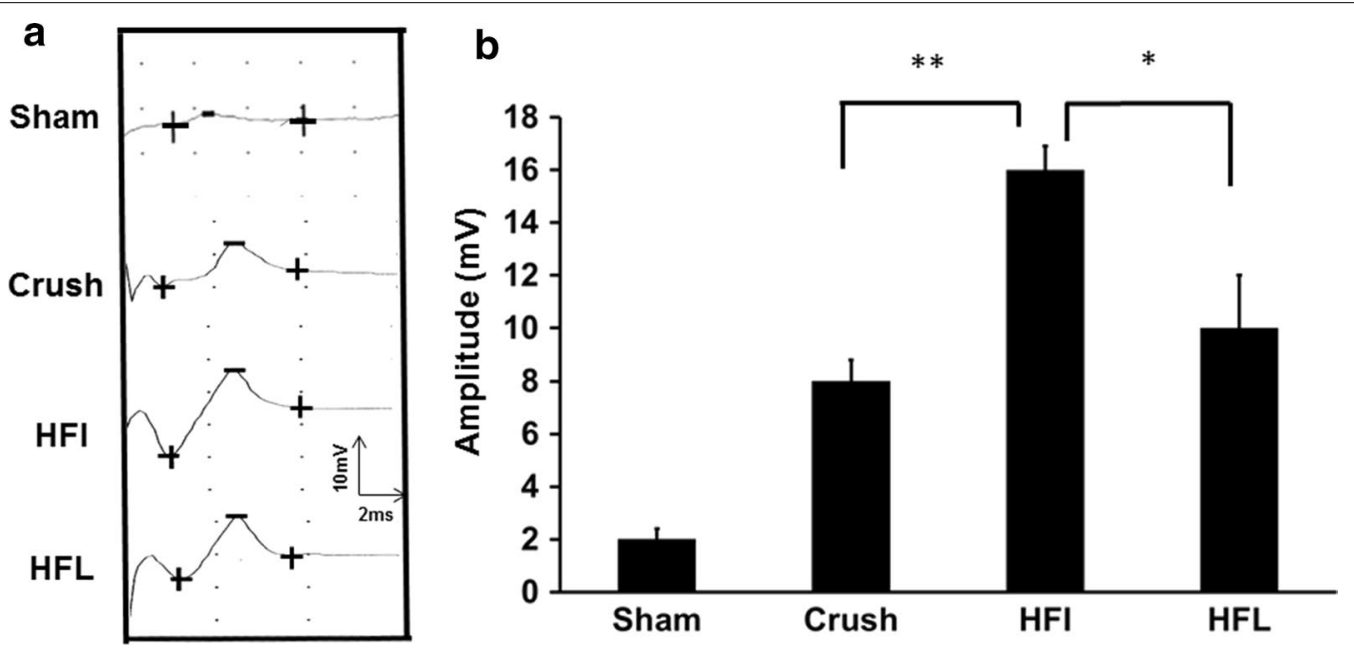

Fig. 6 Somatosensory evoked potential after high-frequency electrical stimulation. a Representative of somatosensory evoked potential in different treatment groups. b Quantitative analysis of somatosensory evoked potential in different treatment groups. Sham, Crush, HFI, HFL: see text; $\mathrm{n}=3 ;{ }^{*} p<0.01,{ }^{* *} p<0.01$ 


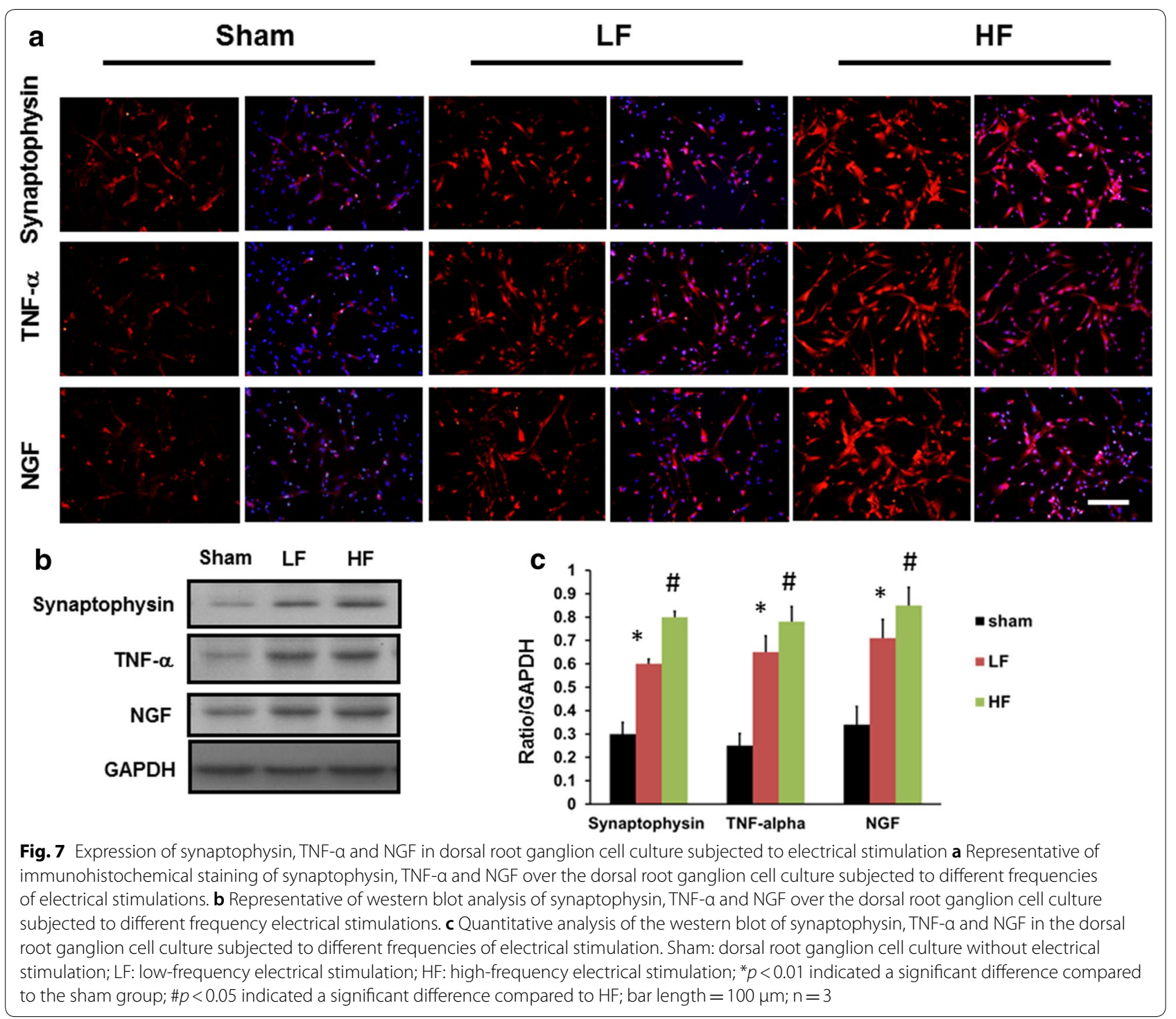

high-frequency electrical muscle stimulation only improved the nerve regeneration without producing an analgesic effect. Furthermore, there was no pain relief following immediate electrical stimulation, however the stimulation predisposed the sensory system to inflammatory changes. Considering the nerve regeneration potential of high-frequency electrical stimulation, a delay in the initiation of stimulation appears advisable.

Dorsal root ganglion cells are located in the intervertebral foramen of the spinal cord and involve the sensory neuron; these cells respond to peripheral nerve injury. The increased expression of TNF- $\alpha$, IL $1 \beta$, and synaptophysin reflected the intensity of the neuropathic pain. The attenuation of these inflammatory cytokines paralleled the decreased neuropathic pain response [20,
21]. In vitro analysis demonstrated that high-frequency electrical stimulation exerted a greater potential for the dorsal root ganglion cells to express an inflammatory response compared to the low-frequency electrical stimulation. In the vivo analysis, immediate high frequency electrical stimulation resulted in a significantly higher expression of inflammatory cytokines in the dorsal root ganglion compared to late high-frequency electrical stimulation and low-frequency electrical stimulation either at the immediate or late time profiles. This result confirmed that a delay in high-frequency electrical stimulation is appropriate to facilitate nerve regeneration without increasing the risk of neuropathic pain.

Electrophysiological and biochemical alterations have been well documented to occur in the somatosensory 
system of the brain after peripheral nervous system injury. An increased expression of TNF- $\alpha$ and synaptophysin in the somatosensory cortex and hippocampus indicate a stronger response to peripheral nerve injury, and an attenuation of this response was noted during a decrease in neuropathic pain [20]. In addition, the evoked potential in the somatosensory cortex was in line with the severity of neuropathic pain with respect to both behavioral and biochemical aspects. This study showed a significantly high amplitude of the evoked potential following immediate high-frequency electrical stimulation. Both electrophysiological and molecular biology data confirmed that immediate high-frequency electrical stimulation had a potential to induce neuropathic pain.

The CatWalk gait XT system is a comprehensive and sensitive tool for the determination of gait alteration in motor and sensory function impairment at the same time profile compared to using the SFI and allodynia analyses alone [20,33]. Motor function improvement is reflected by an increased paw intensity, increased stance, decreased swing and a decreased regularity index. However, sensory impairment is indicated by reciprocal trends [33]. Immediate high-frequency electrical stimulation showed a significant improvement in SFI but decreased the pain threshold in the mechanical allodynia test. The Catwalk gait analysis demonstrated a increased paw density, increased stance, decreased swing and reduced regularity index in motor function accompanying the sensory impairment. In the CatWalk gait analysis, we found that the increased regularity index and decreased paw intensity following immediate high-frequency electrical stimulation was due to the compromised effect of sensory and motor function alterations.

There were some limitations in this study. First, the nerve crush model cannot present the typical sensory impairment observed in well-established neuropathic models such as chronic nerve constriction injury, tibia nerve resection, or nerve root ligation model. However, we aimed to report motor function improvement without aggravation of sensory function through the investigation of different treatment time profiles and the administration of high or low electrical frequency. The nerve-crush injury model consisted of motor and sensory function impairment and was the appropriate model in this study. Second, dorsal root ganglion cell culture, subjected to either high or low-frequency electrical stimulation, did not reflect the status of late TENS biology. However, we intend to investigate the ability of high- and low-frequency electrical stimulation to prime dorsal root ganglion cells to initiate the inflammatory response to increase the nerve regeneration and improve animal behavior after high-frequency electrical stimulation but mitigate the risk of impaired sensory function.

\section{Conclusion}

Both immediate and late administration of highfrequency electrical stimulation exerted a significantly greater motor function improvement than low-frequency electrical stimulation at comparable time points. However, immediate high-frequency electrical stimulation caused a significantly lower pain threshold than late high-frequency or low-frequency stimulation delivered at the immediate or late time points. There, a delay in the initiation of TENS appears to afford a reasonable treatment approach after nerve repair and provides an appropriate time frame for clinical administration.

\section{Additional file}

Additional file 1. The ARRIVE guidelines checklist.

\section{Abbreviations}

TENS: transcutaneous neuromuscular electrical nerve stimulation; NF: neurofilament; SFI: sciatic function index; CMAP: compound muscle action potential; DRGs: dorsal root ganglion cells.

\section{Authors' contributions}

HLS: conception and design of the study, interpretation and acquisition of the immunofluorescence staining, western blot analysis, and writing the manuscript; CYC: study design, acquisition and interpretation of immunofluorescence staining and western blot analysis; ZHL: study design and interpretation of neurobehavior of motor and sensory function; FCC: design and conception of this study as well the interpretation of neurobehavioral analysis such as Catwalk gait analysis and sensory function assessment such as Von-Frey and thermal plate analysis; CJC: Interpretation of immunofluorescence staining and western blot analysis; MLS: conception and design of the study, editing the manuscript and the interpretation of western blot analysis; JS: conception and design of this study and editing the manuscript; HCP: conception and design of this study and approval of the version to be published. All authors read and approved the final manuscript.

\section{Author details}

${ }^{1}$ Department of Life Sciences, Agriculture Biotechnology Center, National Chung-Hsing University, Taichung, Taiwan. ${ }^{2}$ Department of Neurosurgery, Taichung Veterans General Hospital, 1650 Taiwan Boulevard Sec. 4, 40705 Taichung, Taiwan. ${ }^{3}$ Department of Medical Research, Taichung Veterans General Hospital, Taichung, Taiwan. ${ }^{4}$ Institute of Biomedical Sciences, National ChungHsing University, Taichung, Taiwan. ${ }^{5}$ Department of Neurosurgery, University of Virginia, Charlottesville, VA, USA. ${ }^{6}$ Faculty of Medicine, School of Medicine, National Yang-Ming University, Taipei, Taiwan.

\section{Acknowledgements}

The authors would like to thank the research assistant of Miss Shu-Zhen Lai for the kind assistances in the manuscript preparation and the Biostatistics Task Force of Taichung Veterans General Hospital for the statistical analysis.

\section{Competing interest}

The authors declare that they have no competing interests.

\section{Availability of data and materials}

The datasets used and/or analyzed during the current study available from the corresponding author on reasonable request.

Consent for publication

Not applicable. 


\section{Ethics approval and consent to participate}

The ethical approval and consent had been approved by Taichung Veterans General Hospital Institutional Animal Care and Use Committee (IACUC) (Permission No. La-1061455)

\section{Funding}

The authors obtained funding through a grant from Taichung Veterans General Hospital (TCVGH-1054905C) and a joint grant from Taichung Veterans General Hospital/Providence University (TCVGH-PU1048104).

\section{Publisher's Note}

Springer Nature remains neutral with regard to jurisdictional claims in published maps and institutional affiliations.

Received: 14 February 2018 Accepted: 18 June 2018

Published online: 25 June 2018

\section{References}

1. Boncompagni S, Kern H, Rossini K, Hofer C, Mayr W, Carraro U, Protasi F. Structural differentiation of skeletal muscle fibers in the absence of innervation in humans. Proc Natl Acad Sci USA. 2007;104(49):19339-44 Epub 12007 Nov 19327.

2. Kern H, Salmons S, Mayr W, Rossini K, Carraro U. Recovery of long-term denervated human muscles induced by electrical stimulation. Muscle Nerve. 2005;31(1):98-101.

3. Love FM, Son YJ, Thompson WJ. Activity alters muscle reinnervation and terminal sprouting by reducing the number of Schwann cell pathways that grow to link synaptic sites. J Neurobiol. 2003;54(4):566-76.

4. Willand MP, Holmes M, Bain JR, Fahnestock M, De Bruin H. Electrical muscle stimulation after immediate nerve repair reduces muscle atrophy without affecting reinnervation. Muscle Nerve. 2013;48(2):219-25. https://doi.org/10.1002/mus.23726 (Epub 22013 May 23722).

5. Cole BG, Gardiner PF. Does electrical stimulation of denervated muscle, continued after reinnervation, influence recovery of contractile function? Exp Neurol. 1984;85(1):52-62.

6. Gigo-Benato D, Russo TL, Geuna S, Domingues NR, Salvini TF, Parizotto NA. Electrical stimulation impairs early functional recovery and accentuates skeletal muscle atrophy after sciatic nerve crush injury in rats. Muscle Nerve. 2010;41(5):685-93. https://doi.org/10.1002/mus.21549.

7. Marqueste T, Decherchi P, Desplanches D, Favier R, Grelot L, Jammes $Y$. Chronic electrostimulation after nerve repair by self-anastomosis: effects on the size, the mechanical, histochemical and biochemical muscle properties. Acta Neuropathol. 2006;111(6):589-600 (Epub 2006 Mar 2007)

8. Sinis N, Horn F, Genchev B, Skouras E, Merkel D, Angelova SK, Kaidoglou K, Michael J, Pavlov S, Igelmund P. Electrical stimulation of paralyzed vibrissal muscles reduces endplate reinnervation and does not promote motor recovery after facial nerve repair in rats. Ann Anat. 2009;191(4):356-70. https://doi.org/10.1016/j.aanat.2009.1003.1004 (Epub 2009 May 1013).

9. Tam SL, Archibald V, Jassar B, Tyreman N, Gordon T. Increased neuromuscular activity reduces sprouting in partially denervated muscles. J Neurosci. 2001;21(2):654-67.

10. Heidland A, Fazeli G, Klassen A, Sebekova K, Hennemann H, Bahner U. Di lorio B. Neuromuscular electrostimulation techniques: historical aspects and current possibilities in treatment of pain and muscle waisting. Clin Nephrol. 2013;79(Suppl 1):S12-23.

11. Klassen A, Di lorio B, Guastaferro P, Bahner U, Heidland A, De Santo N. High-tone external muscle stimulation in end-stage renal disease: effects on symptomatic diabetic and uremic peripheral neuropathy. J Ren Nutr. 2008;18(1):46-51

12. Strempska B, Bilinska M, Weyde W, Koszewicz M, Madziarska K, Golebiowski T, Klinger M. The effect of high-tone external muscle stimulation on symptoms and electrophysiological parameters of uremic peripheral neuropathy. Clin Nephrol. 2013;79(Suppl 1):S24-7.

13. Schimrigk K, McLaughlin J, Gruninger W. The effect of electrical stimulation on the experimentally denervated rat muscle. Scand J Rehabil Med. 1977;9(2):55-60.
14. Clemente FR, Barron KW. Transcutaneous neuromuscular electrical stimulation effect on the degree of microvascular perfusion in autonomically denervated rat skeletal muscle. Arch Phys Med Rehabil. 1996;77(2):155-60

15. Hennig R. Late reinnervation of the rat soleus muscle is differentially suppressed by chronic stimulation and by ectopic innervation. Acta Physiol Scand. 1987;130(1):153-60.

16. Willand MP, Holmes M, Bain JR, Fahnestock M, de Bruin H. Determining the effects of electrical stimulation on functional recovery of denervated rat gastrocnemius muscle using motor unit number estimation. In: Conference proceedings on IEEE Engineering in medicine and biology society 2011; 2011. p. 1977-80. https://doi.org/10.1109/iembs 2011.6090557

17. Kerns JM, Lucchinetti C. Electrical field effects on crushed nerve regeneration. Exp Neurol. 1992;117(1):71-80.

18. Pan HC, Cheng FC, Chen CJ, Lai SZ, Lee CW, Yang DY, Chang MH, Ho SP. Post-injury regeneration in rat sciatic nerve facilitated by neurotrophic factors secreted by amniotic fluid mesenchymal stem cells. J Clin Neurosci. 2007;14(11):1089-98.

19. Chen CJ, Cheng FC, Su HL, Sheu ML, Lu ZH, Chiang CY, Yang DY, Sheehan J, Pan HC. Improved neurological outcome by intramuscular injection of human amniotic fluid derived stem cells in a muscle denervation model. PLoS ONE. 2015;10(5):e0124624. https://doi.org/10.1371/ journal.pone.0124624 (eCollection 0122015).

20. Chiang CY, Liu SA, Sheu ML, Chen FC, Chen CJ, Su HL, Pan HC. Feasibility of human amniotic fluid derived stem cells in alleviation of neuropathic pain in chronic constrictive injury nerve model. PLoS ONE. 2016;11(7):e0159482. https://doi.org/10.1371/journal.pone.0159482 (eCollection 0152016).

21. Chiang CY, Sheu ML, Cheng FC, Chen CJ, Su HL, Sheehan J, Pan HC. Comprehensive analysis of neurobehavior associated with histomorphological alterations in a chronic constrictive nerve injury model through use of the CatWalk XT system. J Neurosurg. 2014;120(1):25062. https://doi.org/10.3171/2013.9.JNS13353 (Epub 12013 Nov 13351).

22. Cho HY, Suh HR, Han HC. A single trial of transcutaneous electrical nerve stimulation reduces chronic neuropathic pain following median nerve injury in rats. Tohoku J Exp Med. 2014;232(3):207-14.

23. Correa JB, Costa LO, de Oliveira NT, Sluka KA, Liebano RE. Effects of the carrier frequency of interferential current on pain modulation in patients with chronic nonspecific low back pain: a protocol of a randomised controlled trial. BMC Musculoskelet Disord. 2013;14:195. https ://doi.org/10.1186/1471-2474-1114-1195.

24. Suszynski K, Marcol W, Gorka D. Physiotherapeutic techniques used in the management of patients with peripheral nerve injuries. Neural Regen Res. 2015;10(11):1770-2. https://doi.org/10.4103/16735374.170299

25. Willand MP. Electrical stimulation enhances reinnervation after nerve injury. Eur J Transl Myol. 2015;25(4):243-8. https://doi.org/10.4081/ ejtm.2015.5243 (eCollection 2015 Aug 4024)

26. Kao CH, Chen JJ, Hsu YM, Bau DT, Yao CH, Chen YS. High-frequency electrical stimulation can be a complementary therapy to promote nerve regeneration in diabetic rats. PLoS ONE. 2013;8(11):e79078. https ://doi.org/10.71371/journal.pone.0079078 (eCollection 0072013).

27. Chen YS, Hu CL, Hsieh CL, Lin JG, Tsai CC, Chen TH, Yao CH. Effects of percutaneous electrical stimulation on peripheral nerve regeneration using silicone rubber chambers. J Biomed Mater Res. 2001;57(4):541-9.

28. Gordon T, Sulaiman O, Boyd JG. Experimental strategies to promote functional recovery after peripheral nerve injuries. J Peripher Nerv Syst. 2003:8(4):236-50.

29. Robinson AJ. Transcutaneous electrical nerve stimulation for the control of pain in musculoskeletal disorders. J Orthop Sports Phys Ther. 1996;24(4):208-26.

30. Vance CG, Rakel BA, Blodgett NP, DeSantana JM, Amendola A, Zimmerman MB, Walsh DM, Sluka KA. Effects of transcutaneous electrical nerve stimulation on pain, pain sensitivity, and function in people with knee osteoarthritis: a randomized controlled trial. Phys Ther. 2012;92(7):898910. https://doi.org/10.2522/ptj.20110183 (Epub 20112012 Mar 20110130).

31. Carbonario F, Matsutani LA, Yuan SL, Marques AP. Effectiveness of highfrequency transcutaneous electrical nerve stimulation at tender points 
as adjuvant therapy for patients with fibromyalgia. Eur J Phys Rehabil Med. 2013;49(2):197-204 (Epub 2013 Mar 2013).

32. Nnoaham KE, Kumbang J. Transcutaneous electrical nerve stimulation (TENS) for chronic pain. Cochrane Database Syst Rev. 2008;3:CD003222. https://doi.org/10.1002/14651858.CD003222.pub2.
33. Chen YJ, Cheng FC, Sheu ML, Su HL, Chen CJ, Sheehan J, Pan HC. Detection of subtle neurological alterations by the Catwalk XT gait analysis system. J Neuroeng Rehabil. 2014;11:62. https://doi. org/10.1186/1743-0003-1111-1162.

- fast, convenient online submission

- thorough peer review by experienced researchers in your field

- rapid publication on acceptance

- support for research data, including large and complex data types

- gold Open Access which fosters wider collaboration and increased citations

- maximum visibility for your research: over 100M website views per year

At BMC, research is always in progress.

Learn more biomedcentral.com/submissions 